\title{
Sistema inteligente de processamento e análise de vibrações em máquinas rotativas para Manutenção Preditiva Avançada em indústria 4.0
}

\author{
Vinhas PM, Pires Ricardo, Bock EGP, Leão TF* \\ *Instituto Federal de Educação, Ciência e Tecnologia de São Paulo, São Paulo, Brasil.
}

\begin{abstract}
Resumo. A chegada do 5G propicia uma evolução da manutenção preditiva com foco em múltiplos sensores nas máquinas para medir dezenas de parâmetros, garantindo que os dados sejam sempre coletados de forma confiável. Para este objetivo, propõe-se neste trabalho um método adaptativo de aprendizado de máquina para lidar com sinais compostos. O desafio que é lidar com o tamanho variável destes sinais é contornado através da exploração dos parâmetros de um classificador do tipo Máquina de Vetores de Suporte. Alcança-se máxima assertividade na classificação garantindo uma solução adaptativa e eficaz, construída sobre um método consolidado computacionalmente, tornando-se de fácil implementação.
\end{abstract}

Palavras-chave. Análise de vibrações, indústria 4.0, aprendizado de máquina, SVM, 5G.

\section{Intelligent processing and vibration analysis system on rotating machines for Advanced Predictive Maintenance in industry 4.0}

\begin{abstract}
The arrival of 5G provides an evolution of predictive maintenance with a focus on multiple sensors on the machines to measure dozens of parameters, ensuring that data is always collected reliable. For this purpose, an adaptive machine learning method is proposed in this work to deal with composite signals. The challenge of dealing with the varying size of these signals is overcome by exploring the parameters of a support vector machine classifier. Maximum assertiveness in the classification is achieved, guaranteeing an adaptive and effective solution, built on a computationally consolidated method, making it easy to implement.
\end{abstract}

Keywords. Vibration analysis, industry 4.0, machine learning, SVM, 5G. 
Introdução. De acordo com (1), o tempo de inatividade não planejado de um maquinário é um dos maiores problemas para fabricantes, podendo custar até nove vezes mais do que o tempo de inatividade planejado. Isso é particularmente importante para linhas de montagem de alta velocidade, onde o custo da falha em uma máquina pode ser superior a dez mil dólares americanos por minuto. Em média, 4,7\% do tempo de produção planejado é perdido devido ao tempo de inatividade não planejado da máquina parada. Quando o equipamento quebra sem aviso, as implicações são profundas e um processo de reparo é iniciado:

1. Paradas na produção;

2. A equipe de manutenção (no local ou terceirizada) avalia o problema;

3. As peças são encomendadas;

4. Pode ser necessário agendar um especialista para conduzir o reparo no local.

A fim de evitar esses custos, os fabricantes têm usado historicamente visitas de manutenção programadas para garantir que o equipamento esteja em boas condições, mas isso resulta em tempo de inatividade (paradas planejadas para manutenção) e pode resultar em gastos desnecessários (acarretando em custos de manutenção e reparo quando a máquina não exige isso.) A manutenção preditiva existe há vários anos e busca resolver esses problemas. Ao equipar as máquinas com sensores, as condições podem ser monitoradas em tempo real, ou não, e uma combinação de parâmetros são avaliados para determinar quando uma máquina irá quebrar antes do tempo. Isso significa que não apenas o colapso é evitado, mas os gastos com serviços tradicionais são reduzidos e a extensão da vida útil de um ativo é aumentada. Dentre as técnicas utilizadas na manutenção preditiva, uma das mais importantes é a análise de vibrações.

Segundo (1-5), o princípio da análise de vibrações está em que as estruturas das máquinas têm modos de vibração produzidos pela excitação da dinâmica de esforços, cujas frequências são idênticas às dos esforços que os causou, e a soma das respostas vibratórias estruturais correspondem à medida global dos esforços de excitação realizados em algum ponto. A natureza das vibrações mecânicas é caracterizada pelos movimentos oscilatórios em torno de uma posição intermediária.

O atual ambiente da gestão de negócios, caracterizado por um aumento da competição entre as empresas e, simultaneamente, por clientes mais exigentes em relação à qualidade, à tecnologia e ao preço dos bens e serviços, faz com que os gestores busquem novas metodologias e práticas que ajudem no gerenciamento cada vez mais complexo das organizações. Dessa forma, entre as práticas do mundo empresarial, a terceirização estratégica surge pelo fato de as empresas cada vez mais tenderem a se concentrar em suas competências básicas, guardando as energias 
desprendidas em atividades de apoio ou complementares (7). A regra vale para a manutenção preditiva. Grande parte das empresas do setor industrial abrem mão de ter um setor próprio de manutenção preditiva para terceirizar estes serviços. Isto gera uma demanda por profissionais terceirizados entrando e saindo da empresa periodicamente, o que caracteriza uma fuga dos processos naturais da empresa para lidar com estas situações. A problemática é agravada ainda mais pelo fato de as prestadoras de manutenção preditiva serem empresas que, em muitas vezes estão longe da planta industrial contratante, até mesmo em outras regiões geográficas do país, gerando custos de estadia do(s) operador(es) que podem chegar a semanas, dependendo do tamanho da planta industrial e, consequentemente, da quantidade de máquinas a serem monitoradas.

O termo rede industrial refere-se à implementação de protocolos de comunicação entre equipamentos de campo, controladores digitais (CLPs), conjuntos de software e também conexão com sistemas externos. São altamente especializadas e fazem uso de uma variedade de protocolos adaptados para cumprir os requisitos rigorosos do controle e monitoramento em tempo real de equipamentos. Com os avanços recentes em redes industriais, como a incorporação da tecnologia Ethernet, começou-se a estreitar a linha entre redes industriais e comerciais. Em seus núcleos, cada uma delas tem requisitos fundamentalmente diferentes (8).

De acordo com $(8,9)$, a diferença mais essencial é que as redes industriais são conectadas a equipamentos físicos de alguma forma e são usadas para controlar e monitorar ações e condições do mundo real. Isso resultou na ênfase em um conjunto diferente de considerações de qualidade de serviço (QoS) para as redes comerciais como a necessidade de forte determinismo e transferência de dados em tempo real.

Do ponto de vista de redes industriais, as fábricas têm historicamente usado redes fixas (ethernet) para se conectar, o que leva a uma significativa quantidade de tempo e custo de implantação. Também significa que os fabricantes em mercados sem a infraestrutura fixa não têm sido capazes de avançar seus processos de fabricação tão rapidamente. $\mathrm{O}$ 5G oferece uma alternativa à rede tradicional, pois tem a capacidade de transportar tanto largura de banda como redes fixas em velocidades altas o suficiente. Isso é particularmente benéfico para aqueles fabricantes em países com infraestrutura fixa limitada, mas com infraestrutura de rede móvel relativamente madura. Eles agora poderão usar o acesso fixo sem fio $5 \mathrm{G}$ e a banda larga, sem a necessidade de instalar cabos. Isso permitirá que os fabricantes que têm sido mais lentos na evolução tecnológica progridam na digitalização para dar um salto para a Indústria 4.0 (1).

A indústria 4.0, ou a quarta revolução industrial tem como fundamento básico conectar máquinas, sistemas e ativos, podendo criar redes inteligentes e assim controlar os módulos de 
produção de forma autônoma. Ela permite resolver a necessidade de estar na planta com frequência de forma efetiva e menos custosa financeiramente para o contratante e para a empresa de manutenção preditiva contratada. Mas, gera novos gargalos.

Tecnologias sem fio atuais como ZigBee, WirelessHART e ISA 100.11a, que são baseadas nos padrões IEEE 802.15, são empregadas, principalmente, para aplicações de monitoramento. O mesmo ocorre com as redes locais sem fio baseadas em IEEE 802.11 (WLANs), também conhecidas como redes Wi-Fi, que oferecem vários benefícios para a comunicação industrial. Dentre as soluções baseadas na IEEE 802.15, o Wi-Fi é a que oferece melhor cobertura e suporta taxas de dados mais altas (10). Ainda assim, o 5G é particularmente mais atraente para a comunicação industrial devido a uma série de fatores:

Interface sem fio unificada - $5 \mathrm{G}$ oferece uma interface sem fio unificada, uma interface para suportar os diversos requisitos de diferentes aplicações industriais. Ele foi projetado para três principais categorias de serviço $(11,12)$ : banda larga móvel aprimorada (eMBB) com taxa de dados de pico de até $10 \mathrm{Gbps}$, comunicações massivas entre máquinas, (mMTC) com densidade de conexão de até até 1 milhão de conexões por metro quadrado e comunicações de baixa latência ultra confiável (uRLLC) fornecendo latência de plano do usuário de 1 milissegundo (ms) com mais de 99,999\% de confiabilidade. Essa capacidade não é oferecida por nenhuma outra tecnologia sem fio industrial existente.

Qualidade de serviço garantida (QoS) - ao contrário das tradicionais tecnologias sem fio industriais baseadas em Wi-Fi ou Bluetooth, o 5G fornece QoS garantido para aplicações industriais críticas.

Mobilidade - plataformas móveis como veículos guiados automatizados e robôs móveis são uma característica fundamental de vários sistemas emergentes e futuros sistemas industriais $(11,13)$. O 5G fornece suporte integrado para manipulação de mobilidade em ambientes industriais.

Segurança - o 5G traz uma tecnologia de segurança comprovada e testada para o mundo industrial que foi implantada em redes de celular ao redor do mundo.

Posicionamento - capacidades de posicionamento, que receberam atenção significativa nas gerações anteriores, estão sob consideração para 5G. Uma avaliação inicial dos requisitos de posicionamento revelam que as metas são bastante altas (por exemplo, precisão dentro de $10 \mathrm{~cm}$ com latência de menos do que $15 \mathrm{~ms}$ ) (11,13). Tais recursos de posicionamento de alta precisão seriam cruciais para muitas aplicações industriais emergentes. 
A recente tecnologia IEEE 802.11ax (Wi-Fi 6) é um concorrente potencial do 5G. Ele fornece uma latência muito menor em comparação com as gerações anteriores de Wi-Fi, o que o torna atraente para aplicações industriais $(11,15)$. No entanto, $5 \mathrm{G}$ tem um vantagem distinta sobre Wi-Fi 6 para comunicação industrial, devido a uma série de fatores, como desenho de interface aérea flexível, suporte nativo para uRLLC e mobilidade, garantias de QoS e melhor escalabilidade (11).

A manutenção preditiva e como se agrega a concepção de internet das coisas a ela é um conceito até mesmo intuitivo, mas os benefícios não se materializaram da forma como foram teorizados. Em parte, isso ocorre, segundo (1), porque houve desafios para integrar percepções de tecnologia operacional em sistemas de TI (por exemplo, sistemas ERP). Outros problemas decorrem da incapacidade de prever os resultados de forma eficaz porque não há variáveis suficientes sendo medidas e as plataformas de aprendizado de máquina não estão maduras o suficiente para uma modelagem real do problema.

O 5G suportará uma nova onda de manutenção preditiva intitulada por (1) como "Manutenção Preditiva Avançada". Isso significa aumentar o número de sensores instalados no equipamento de um ou dois para medir dezenas de parâmetros - temperatura, vibrações, umidade, pressão, etc. - e garantindo que os dados sejam sempre coletados de forma confiável em tempo real. Isso é importante, porque o valor vem desde a análise dos dados, cruzando-os com dados históricos e tendências para prever resultados de forma proativa. Os dados precisam ser os mais completos possíveis para garantir que as anomalias não sejam perdidas, como pode ser um único ponto de dados que detecta o problema e determina se a máquina precisa ser reparada.

Entre os fenômenos que podem causar picos no espectro cujas frequências são ligadas ao rotor podem-se indicar: desequilíbrio, desalinhamento, mau ajuste mecânico e danos. O fenômeno de desequilíbrio é a causa mais comum de vibrações. Por exemplo, é impossível na construção de um rotor equilibrá-lo perfeitamente; apesar do cuidado com que é construído, sempre haverá uma vibração durante a rotação cuja amplitude é diretamente proporcional à magnitude do desequilíbrio e ao quadrado da magnitude da rotação $(2,4,6)$.

Técnicas no domínio do tempo e frequência são usadas principalmente para processar sinais vibratórios não estacionários. Entre as mais eficientes, está a Transformada de HilbertHuang, que consiste na aplicação de duas técnicas: o EMD (Empirical Mode Decomposition) e a Transformada de Hilbert. O EMD identifica os modos oscilatórios intrínsecos, ou seja, as funções de modo intrínseco (IMFs) do sinal, e então, a Transformada de Hilbert é aplicada a cada IMF para extrair as frequências e amplitudes instantâneas. Como resultado, uma representação 
tridimensional (amplitude, frequência, tempo) de cada modo oscilatório do sinal é obtida. $(16,17)$.

De acordo com $(18,19)$, o EMD padrão usa um método de interpolação para calcular o valor médio da curva de envelope superior e inferior do sinal univariável de forma a obter o valor médio local. No entanto, o valor do máximo local e do mínimo não pode ser definido diretamente, e com isso as IMF extraídas não são claras. Para abordar essas questões, o EMD multivariável foi desenvolvido. Este método considera o sinal n-variável (n2) como uma série temporal $\mathrm{N}$-dimensional e escolhe os vetores de direção apropriados no espaço $\mathrm{N}$-dimensional. As $\mathrm{N}$-variáveis do sinal são respectivamente projetadas na direção dos vetores selecionados para formar o sinal de projeção e calcular todos os envelopes de cada sinal projetado, finalmente calculando a média de todos aqueles para obter a média local do sinal multivariável. Então, seguindo as etapas do EMD padrão, todos os grupos de IMFs podem ser calculados, alcançando assim a decomposição EMD multivariáveis do sinal multivariável original.

A aprendizagem de máquina na forma da máquina de vetores de suporte (SVM) tem uma base teórica bem estabelecida e interpretação geométrica intuitiva. É amplamente aplicada, servindo como base em visão computacional, reconhecimento de padrões, recuperação de informações, e mineração de dados, etc $(16,20)$.

O objetivo deste artigo é propor um método adaptativo de classificação de falhas de rolamentos para ser empregado na manutenção preditiva avançada no contexto da indústria 4.0 e da quinta geração da telefonia móvel, o 5G, ou seja, que possa ser utilizado com sinais compostos, formados por outros sinais. Por exemplo, provenientes de múltiplos sensores e que esta quantidade de sensores seja variável.

Metodologia adotada para o diagnóstico das falhas nos rolamentos. Neste trabalho, é proposta uma metodologia que envolve três passos principais: extração das características, seleção das características e classificação. Após a aquisição dos dados de vibração, executa-se a decomposição de modo empírico (EMD) para extrair as IMFs do sinal, e selecionam-se as mais importantes de acordo com a maior correlação com o sinal original. Os dados são então divididos em dois grupos para treino e teste da SVM multiclasses.

Em seu tipo mais simples, a SVM (21) não oferece suporte à classificação multiclasse nativamente. Ele oferece suporte à classificação binária e à separação de pontos de dados em duas classes. Para classificação multiclasse, o mesmo princípio é utilizado depois de decompor o problema de multiclassificação em vários problemas binários de classificação. A ideia é mapear pontos de dados para espaços dimensionais elevados para obter separação linear mútua entre 
cada par de classes. Isso é chamado de abordagem Um-para-Um, que divide o problema multiclasse em vários problemas de classificação binária, com um classificador binário para cada par de classes. Outra abordagem que pode ser usada é "um-contra-todos", utilizada neste trabalho.

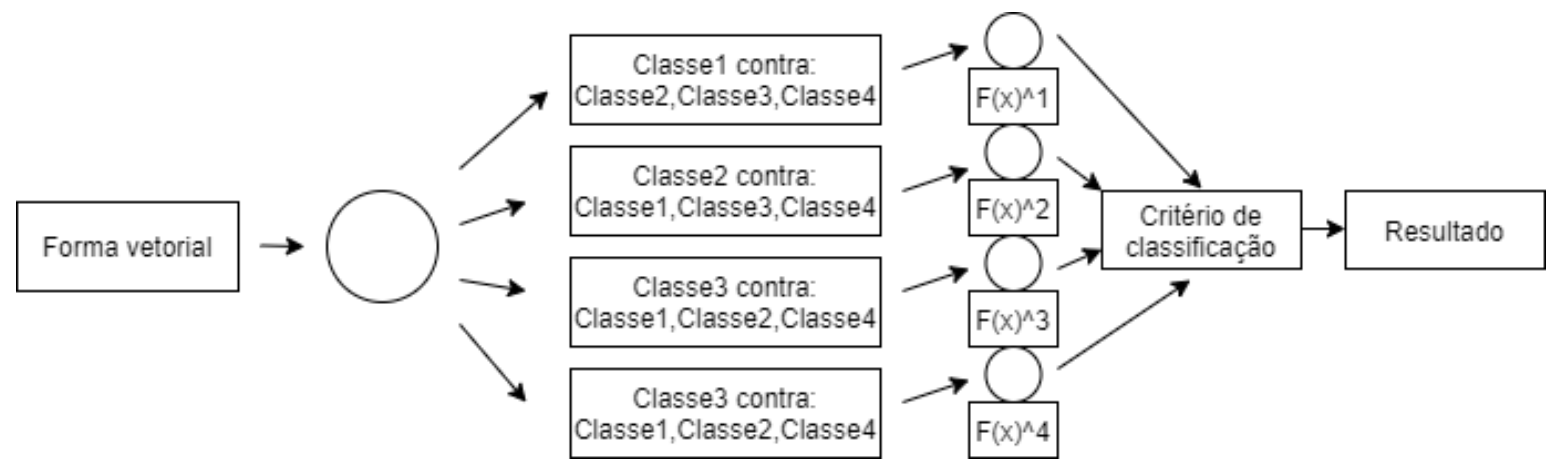

Figura 1 - Diagrama esquemático do algorítmo de classificação SVM Um-contra-todos (1).

Análise de série não linear no domínio do tempo-frequência. As características não estacionárias e não lineares dos sinais de vibração em rolamentos são percebidas na Figura 2. Elas são a justificativa para a aplicação do modo de decomposição empírico para a condução deste trabalho.
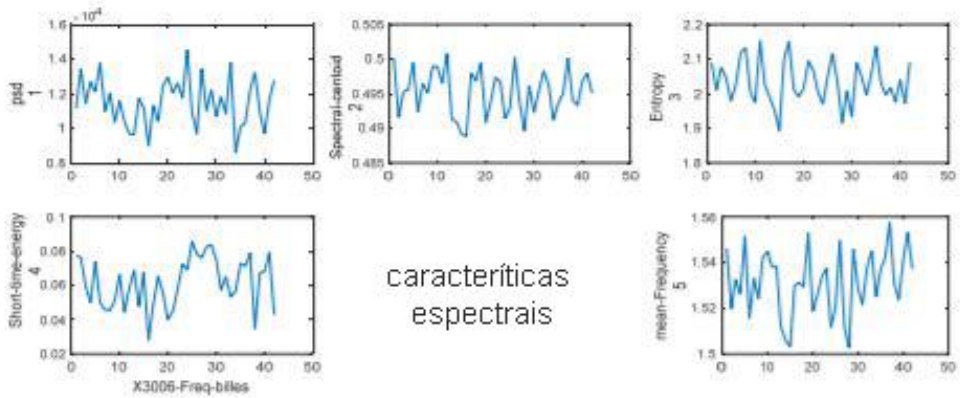

caracteriticas espectrais
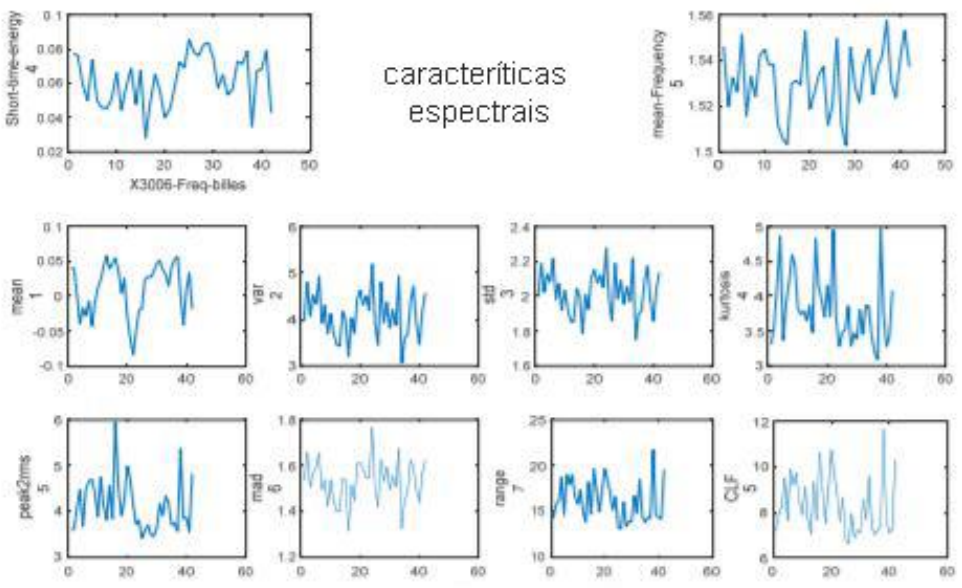

caracteríticas

temporais

Figura 2 - Análise das características não estacionárias da esfera do rolamento com defeito de diâmetro de 0.028 ", em motor a vazio, a $1772 \mathrm{rpm}$, no tempo e na frequência $(1,26)$. 
Para composição dos vetores de características foram selecionadas as seguintes funções matemáticas no domínio do tempo:

Média, variância, kurtosis, fator de folga, desvio médio absoluto, intervalo, desvio padrão e no domínio da frequência:

Frequência média, frequência média da potência, densidade espectral da potência, entropia espectral.

Todas as funções estão descritas em função da amplitude no ponto de amostragem $\mathrm{N}$ em (1).
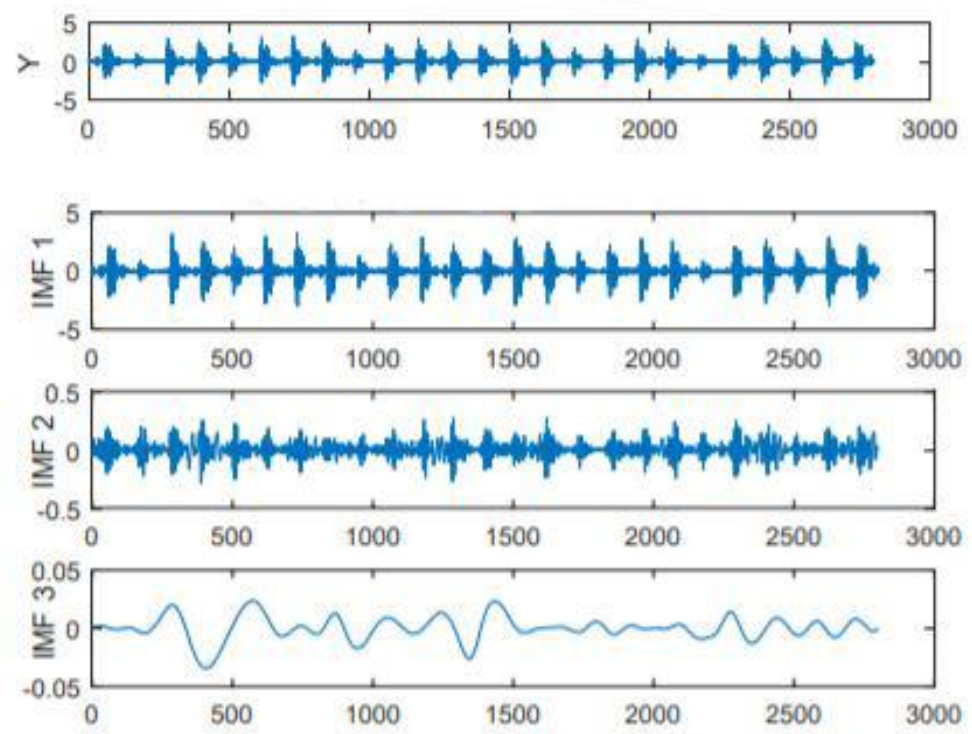

Figura 3 - Sinal original (Y) e as três primeiras IMFs da pista externa do rolamento com defeito de diâmetro de 0.007”, em motor a vazio, a 1797 rpm $(1,26)$.

Os espectros têm picos que permitem identificar falhas. Da avaliação das frequências de defeito dos rolamentos de acordo com o banco de dados da Case Western University e das especificações da plataforma de teste por eles utilizadas, destacam-se os seguintes valores:

- A frequência de falha da pista interna é 160 162,1 Hz;

- A frequência da falha da pista externa é 106 107,3 Hz;

- A frequência de falha de esferas é $139 \sim 141,1 \mathrm{~Hz}(16,22,23)$. 
Análise de fator de correlação de falha. Quando se escolhe utilizar a decomposição por modo intrínseco, pressupõe-se que todos os dados consistem de diferentes e simples modos intrínsecos de oscilações. Cada modo intrínseco, que pode ser linear ou não, representa uma oscilação simples, que contém o mesmo número de extremos e cruzamentos em zero. (24). Sendo assim, para dados cujos modos intrínsecos são não estacionários, mas com larguras de banda bem definidas e não sobrepostas, as abordagens EMD são capazes de produzir IMFs significativas (25). Isso dá margens para que trabalhos anteriores (1) realizassem a escolha das IMFs mais próximas do sinal original baseados numa correlação visual, como pode ser visto na Figura 3 , sem comprometer a qualidade do trabalho. Para um sinal composto com quantidade de componentes variável, a correlação não fica visível e precisa de um método matemático para ser estabelecida. Vale ressaltar que estes componentes precisam ter uma relação mínima, como por exemplo, serem provenientes de diferentes sensores posicionados em diferentes locais da uma mesma máquina, como na Figura 2.

Neste trabalho, buscou-se avaliar a possibilidade de contornar essa necessidade, pois, essa correlação pode aumentar o estágio de pré-processamento dos dados, como também ser custoso para a unidade de processamento, causando restrições na escolha do hardware onde devem ser implantadas. Ponto relevante no contexto da indústria 4.0.

Foram feitos dois processos de extração das IMFs que estão detalhados à frente na Tabela 3 como resultados deste trabalho. Em seguida, a partir da(s) IMF(s) mais relevante(s) extraem-se os parâmetros temporais e espectrais, os quais comporão os vetores de características para cada classe.

Os dados resultantes são divididos em duas partes: uma parte para a etapa de classificação do SVM multiclasses e outra parte para os testes.
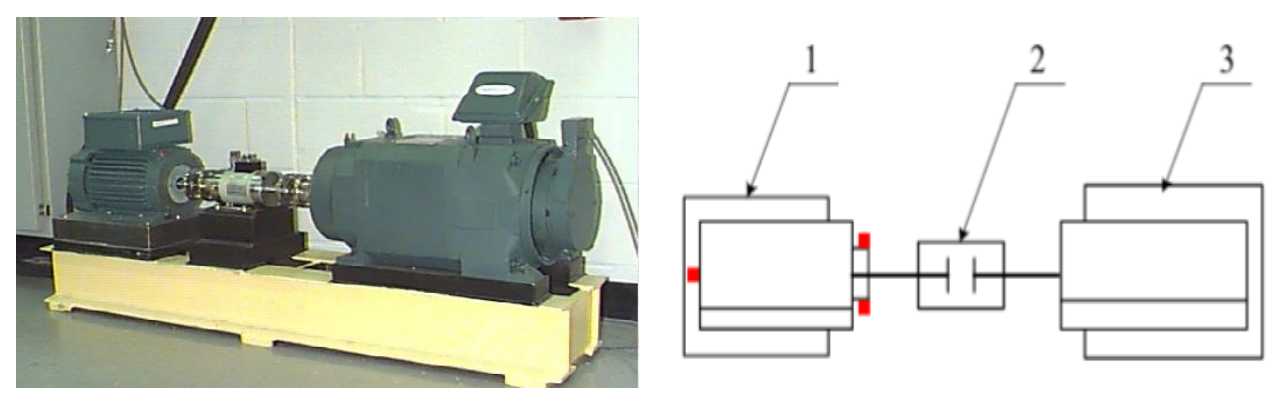

Figura 4 (a) - Equipamento de testes da Case Western University (26).

(b) - Diagrama esquemático do motor (18). Em vermelho, os locais onde os sensores foram posicionados. 1- Motor de 2HP. 2- Transdutor de torque. 3- Dinamômetro. 
Aplicação do método sobre o banco de dados da Case Western University. O trabalho foi conduzido sobre a base de dados da Case Western University com sinais de máquinas em bom estado, de máquinas com diferentes tipos de falhas e medidos em pontos diferentes da máquina rotativa.

O experimento no qual o banco de dados é gerado usou um motor a indução trifásico de dois cavalos de potência, um transdutor de torque e um dinamômetro no eixo do motor como mostrado na Figura 5. Na extremidade de acionamento, existe um rolamento de esferas 62052RS JEM SKF, no qual acelerômetros com um largura de banda de até $5000 \mathrm{~Hz}$ foram instalados. Os dados foram amostrados a uma frequência de $12 \mathrm{KHz}$.

Os experimentos daquele trabalho foram conduzidos em rolamento de extremidade para os casos de normalidade, defeito da pista interna, defeito da pista externa e defeito de esferas, com o gravidade dos defeitos da seguinte forma:

1. Diâmetro do defeito da pista interna: 0,014".

2. Diâmetro do defeito da pista externa: 0,007".

3. Diâmetro do defeito da esfera 0,028".

As simulações deste trabalho foram conduzidas nas seguintes especificações:

Tabela 2. Ambiente

\begin{tabular}{|l|l|}
\hline Nome do sistema operacional & Microsoft Windows 10 Pro \\
\hline Versão do sistema operacional & 10.0 .19042 N/A compilação 19042 \\
\hline Arquitetura do sistema operacional & 64 bits \\
\hline Processador & $\begin{array}{l}\text { Intel(R) Core(TM) i7-4510U CPU @ 2.00GHz } 2.60 \\
\text { GHz }\end{array}$ \\
\hline Memória & $8 \mathrm{~GB}$ \\
\hline Linguagem & Python 3.9.2 \\
\hline Bibliotecas & Scipy, Numpy, Matplotlib, PyEMD, Scikit-learn \\
\hline
\end{tabular}


Tabela 3. Resultados

\begin{tabular}{|c|c|c|c|c|c|c|c|c|c|}
\hline \multicolumn{5}{|c|}{ Pré-processamento } & \multicolumn{3}{|l|}{ SVM } & & \multirow[b]{2}{*}{$\begin{array}{l}\text { Acurácia } \\
(\%)\end{array}$} \\
\hline $\begin{array}{l}\text { Qtde de } \\
\text { compon } \\
\text { entes } \\
\text { do sinal }\end{array}$ & $\begin{array}{l}\text { Qntd } \\
\text { e de } \\
\text { IMF } \\
\text { s }\end{array}$ & $\begin{array}{l}\text { Qtde. } \\
\text { de } \\
\text { caracter } \\
\text { ísticas }\end{array}$ & $\begin{array}{l}\text { Uso } \\
\text { de } \\
\text { filtro }\end{array}$ & $\begin{array}{l}\text { amostras/ } \\
\text { janelas } \\
\text { de treino/ } \\
\text { teste }\end{array}$ & $\begin{array}{l}\text { param } \\
. \mathrm{C}\end{array}$ & $\begin{array}{l}\text { param } \\
\text { Gama }\end{array}$ & $\begin{array}{l}\text { Algoritmo de } \\
\text { normalização }\end{array}$ & $\begin{array}{l}\text { Tempo } \\
\text { (s) }\end{array}$ & \\
\hline 4 & 1 & 11 & Não & $\begin{array}{l}2048 / 30 / \\
13\end{array}$ & 1.0 & $\begin{array}{l}1 / \mathrm{Nxv} \\
\operatorname{ar}(\mathrm{n})^{1}\end{array}$ & $\begin{array}{l}\text { máx. } \\
\text { absoluto }\end{array}$ & 40,705 & 100 \\
\hline 9 & 1 & | / & Não & / & / & / & $\begin{array}{l}\text { máx. } \\
\text { absoluto }\end{array}$ & 76,891 & 80,34 \\
\hline 9 & 2 & | / & Não & / & I & I & $\begin{array}{l}\text { máx. } \\
\text { absoluto }\end{array}$ & 81,282 & 83,76 \\
\hline 9 & 3 & | / & Sim & / & / & / & $\begin{array}{l}\text { máx. } \quad \text { valor } \\
\text { absoluto }\end{array}$ & 105,202 & 88,03 \\
\hline 9 & 3 & | / & Não & / & / & 1 & $\begin{array}{l}\text { máx. } \\
\text { absoluto }\end{array}$ & 105,162 & 88,03 \\
\hline 9 & 3 & | / & Não & / & 10 & 1 & $\begin{array}{l}\text { máx. } \\
\text { absoluto }\end{array}$ & 105,172 & 100 \\
\hline \multicolumn{10}{|l|}{ Legenda: } \\
\hline \multicolumn{10}{|c|}{$\begin{array}{l}\text { Azul: Sinal composto com quantidade de componentes fixa. } \\
\text { Verde: Sinais compostos com quantidade de componentes variáveis. } \\
\text { 1: Sendo n a amplitude no ponto de amostragem. }\end{array}$} \\
\hline
\end{tabular}

De acordo com a tabela 3, um resultado melhor foi alcançado para um sinal composto de tamanho fixo, como era esperado. Para sinais compostos de tamanhos distintos, conseguiu-se alcançar cem por cento de assertividade contornando a correlação entre as multivariáveis, o 
objetivo deste trabalho, alterando os parâmetros da SVM, sem custo adicional de tempo. A diferença de $>60$ s no processo é natural devido ao aumento de variáveis. Uma observação a se fazer é a indiferença na filtragem do sinal.

Conclusão. Conclui-se que é possível e sensato utilizar o EMD para trabalhar com sinais compostos formados por quantidades de componentes distintas no contexto da manutenção preditiva avançada, onde, embasada pelo pilar das comunicações massivas entre máquinas, do 5G (mMTC), que pode suportar uma densidade de conexão extremamente alta de dispositivos conectados, endossa o uso de múltiplos sensores nas máquinas para cruzar dados a fim de gerar informações mais precisas sobre o estado da máquina. $\mathrm{O}$ cem por cento de acurácia confirma $\mathrm{o}$ sucesso na classificação, garantindo o objetivo deste trabalho. Isso se traduz em casos reais na indústria em, por exemplo, ficando a cargo do arquiteto da solução, distribuir a quantidade de sensores conforme as particularidades do projeto e sem se preocupar em ter quantidades de amostras proporcionais às classes para a etapa de classificação, podendo até mesmo treinar a máquina com o auxílio de mais de um banco de dados públicos e confiáveis. Uma grande vantagem deste método é ser construído sobre uma abordagem matemática consolidada do ponto de vista computacional. Existem excelentes bibliotecas a serem utilizadas em diferentes linguagens de programação como Python e $\mathrm{C}++$.

Reconhecimentos. Os reconhecimentos pelo suporte são destinados ao Instituto Federal de São Paulo.

Divulgação. Os autores declaram não haver conflitos de interesse neste trabalho. Estes também endossam a iniciativa de ciência aberta. Os códigos das simulações feitas neste artigo e detalhes do processo podem ser encontrados sob licença GPL 3.0 em https://1000bbits.me/scientificpaper-intelligent-processing-and-vibration-analysis-system-on-rotating-machines-for-advancedpredictive-maintenance-in-industry-4-0/.

\section{Referências}

(1) Adib, D. 5G's Impact on Manufacturing-\$740 BN of Benefits in 2030. In: STL Partners. 2019. 
(2) Neto SS, Sobrinho JRCS, Costa CD, Leão TF, Senra SAMM, Bock EGP, et al. Investigation of MEMS as accelerometer sensor in an Implantable Centrifugal Blood Pump prototype. Journal of the Brazilian Society of Mechanical Sciences and Engineering. 2020;42(9).

(3) Meirovitch L. Fundamentals of vibrations. Waveland Press; 2010.

(4) Mirshawka, Victor. Manutenção Preditiva-Caminho para zero defeitos. McGrawHill; São Paulo, 1991.

(5) Norton MP, Karczub DG. Fundamentals of noise and vibration analysis for engineers. Cambridge University Press; 2003.

(6) Kardec, A., and JA NASCIF. Manutenção Preditiva: fator de sucesso na gestão empresarial. Rio de Janeiro, Brasil. 2013:196.

(7) Leocádio L, Dávila GA, Varvakis G. Evolução da terceirização estratégica diante da gestão por processo. Anais, 11 Simpósio de Administração da Produção, Logística e Operações Internacionais. 2008.

(8) Galloway B, Hancke GP. Introduction to Industrial Control Networks. IEEE Communications Surveys \& Tutorials. 2013;15(2):860-80.

(9) Stouffer K, Falco J, Scarfone K. Guide to Industrial Control Systems (ICS) security : Supervisory Control and Data Acquisition (SCADA) systems, Distributed Control systems (DCS), and other control system configurations such as Programmable Logic Controllers (PLC) : recommendations of the National Institute of Standards and Technology, Computer security. 2011.

(10) Aijaz A. High-Performance Industrial Wireless: Achieving Reliable and Deterministic Connectivity Over IEEE 802.11 WLANs. IEEE Open Journal of the Industrial Electronics Society. 2020;1:28-37.

(11) Aijaz A. Private 5G: The Future of Industrial Wireless. IEEE Industrial Electronics Magazine. 2020;14(4):136-45.

(12) Series, M. IMT Vision-Framework and overall objectives of the future development of IMT for 2020 and beyond. Recommendation ITU. 2015 Sep;2083.

(13) Aijaz A, Sooriyabandara M. The Tactile Internet for Industries: A Review. Proceedings of the IEEE. 2019;107(2):414-35.

(14) 3GPP, "Study on Positioning Use cases Stage 1 (Release 16)," 3rd Generation Partnership Project (3GPP), TR 22.872, Sept. 2018, v16.1.0. [Online]. Disponível em: https://www.3gpp.org/ftp/Specs/archive/22 series/ 22.872/

(15) Aijaz A. High-Performance Industrial Wireless: Achieving Reliable and Deterministic Connectivity Over IEEE 802.11 WLANs. IEEE Open Journal of the Industrial Electronics Society. 2020;1:28-37. 
(16) Korba KA, Arbaoui F. SVM Multi-Classification of Induction Machine's bearings defects using Vibratory Analysis based on Empirical Mode Decomposition. International Journal of Applied Engineering Research. 2018;13(9):6579-86.

(17) Lei Y, Lin J, He Z, Zuo MJ. A review on empirical mode decomposition in fault diagnosis of rotating machinery. Mechanical systems and signal processing. 2013 Feb 1;35(1-2):108-26.

(18) Lv Y, Yuan R, Song G. Multivariate empirical mode decomposition and its application to fault diagnosis of rolling bearing. Mechanical Systems and Signal Processing. 2016 Dec 15;81:219-34.

(19) Deléchelle E, Lemoine J, Niang O. Empirical mode decomposition: an analytical approach for sifting process. IEEE Signal Processing Letters. 2005 Oct 17;12(11):764-7.

(20) Wu SD, Wu PH, Wu CW, Ding JJ, Wang CC. Bearing fault diagnosis based on multiscale permutation entropy and support vector machine. Entropy. 2012 Aug;14(8):1343-56.

(21) Cortes C, Vapnik V. Support-vector networks. Machine learning. 1995 Sep;20(3):273-97.

(22) Raj AS, Murali N. Morlet wavelet UDWT denoising and EMD based bearing fault diagnosis. Electronics. 2013 Jun 15;17(1):1-8.

(23) Wei Z, Gao J, Zhong X, Jiang Z, Ma B. Incipient fault diagnosis of rolling element bearing based on wavelet packet transform and energy operator. WSEAS Transactions on Systems. 2011 Mar 1;10(3):81-90.

(24) Chaudhari H, Nalbalwar SL, Sheth R. A review on intrensic mode function of EMD. In2016 International Conference on Electrical, Electronics, and Optimization Techniques (ICEEOT) 2016 Mar 3 (pp. 2349-2352). IEEE.

(25) Mandic DP, ur Rehman N, Wu Z, Huang NE. Empirical mode decomposition-based timefrequency analysis of multivariate signals: The power of adaptive data analysis. IEEE signal processing magazine. 2013 Oct 16;30(6):74-86.

(26) Loparo KA. Bearing vibration data set. https://csegroups.case.edu/bearingdatacenter /pages/welcome-case-western-reserve-university-bearing-data-center-website

\section{Authors ORCID}

Paulo Matheus Vinhas - https://orcid.org/0000-0003-0891-6649

Ricardo Pires - http://orcid.org/0000-0003-4677-8435

Eduardo Bock - https://orcid.org/0000-0003-3962-9052

Tarcísio Leão - http://orcid.org/0000-0003-4884-5638 